\title{
Electrostatic Tuning of the Bending Stiffness of a large scale GFRP-CFRP Beam
}

\author{
Bergamini A., Christen R. and Motavalli M. \\ Empa Swiss Federal Laboratories for Materials Testing and Research, 129 Ueberlandstrasse, \\ CH- 8600 Duebendorf, Switzerland;
}

\begin{abstract}
The suppression of vibrations of a structure is commonly considered a necessary measure for the extension of its lifetime, when high amplitude vibrations are observed. As an alternative to the introduction of discrete damping devices, the modification of the stiffness of a beam is proposed as a means to suppress vibrations due to resonance, thank to the ability to reject mechanical energy input at specific frequencies. Previous work has outlined the principle and the potential advantages of such an approach based on the behavior of a small scale system. In order to confirm the feasibility of the approach on macro-scale systems, such as a light weight pedestrian bridge, experiments for the tuning of a $2.5 \mathrm{~m}$ long glass fiber reinforced polymer I-beam were performed. The results of the experiments show that it is possible to modify the bending stiffness of structural elements that can be used for real life engineering applications. Measurements show that it is possible to shift the resonance peak of a beam while maintaining a reasonably good q-factor in the transfer function, thus indicating that the change in behavior happens in connection with an increased stiffness rather than with the introduction of substantial damping. Based on the presented feasibility study, the development of an adaptive bridge deck will be considered.
\end{abstract}

Keywords: Adaptive system, bending stiffness, tuning, multilayer structures

\section{INTRODUCTION}

In 2003, Empa's Structural Engineering Research Laboratory designed and built a full scale modular lightweight pedestrian cable stayed bridge $^{1}$ that is used as a research platform to investigate novel structural health monitoring systems as well as vibration mitigation methods. The bridge deck is made of pultruded glass fiber reinforced polymer (GRFP) elements, see figure 1. The structure lends itself as an outstanding demonstrator for the efficacy of the modification of the bending stiffness of structural elements as an approach to the mitigation of the vibration of lightweight structures.
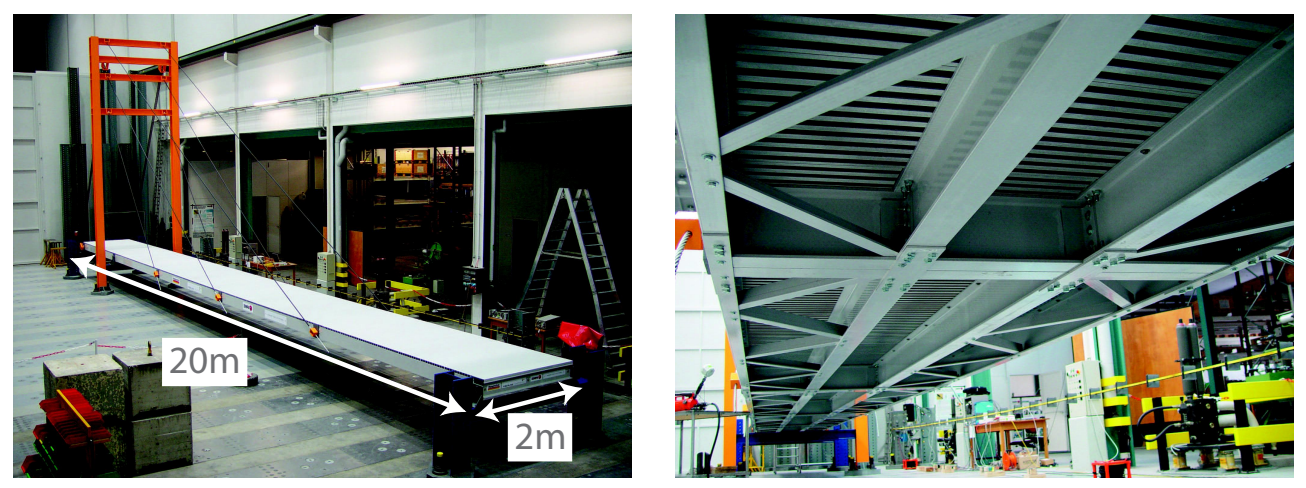

Figure 1. Empa's full scale lightweight pedestrian bridge is used as a test platform for structural health monitoring and vibration mitigation developments. Left, general view. Right, detail from the bottom

Further author information:

Andrea Bergamini: E-mail: andrea.bergamini@empa.ch, Telephone: +41 448234424

Smart Structures and Materials 2006: Damping and Isolation, edited by William W. Clark, Mehdi Ahmadian, Arnold Lumsdaine, Proc. of SPIE Vol. 6169, 616919, (2006) · 0277-786X/06/\$15 · doi: 10.1117/12.659207 
In this contribution, the main results of a feasibility study for the modification of the mechanical properties of the load bearing elements of the bridge deck are presented. For this purpose, a $2.5 \mathrm{~m}$ long GRFP I-beam was modified so as to allow for the electrostatic mechanical coupling of additional stiffening elements consisting of carbon fiber reinforced polymer plates using commercially available materials. The material properties of the beam used for the experiments closely match the ones of the elements the bridge deck is made of, while its cross section has smaller dimensions, as shown in figure 2:

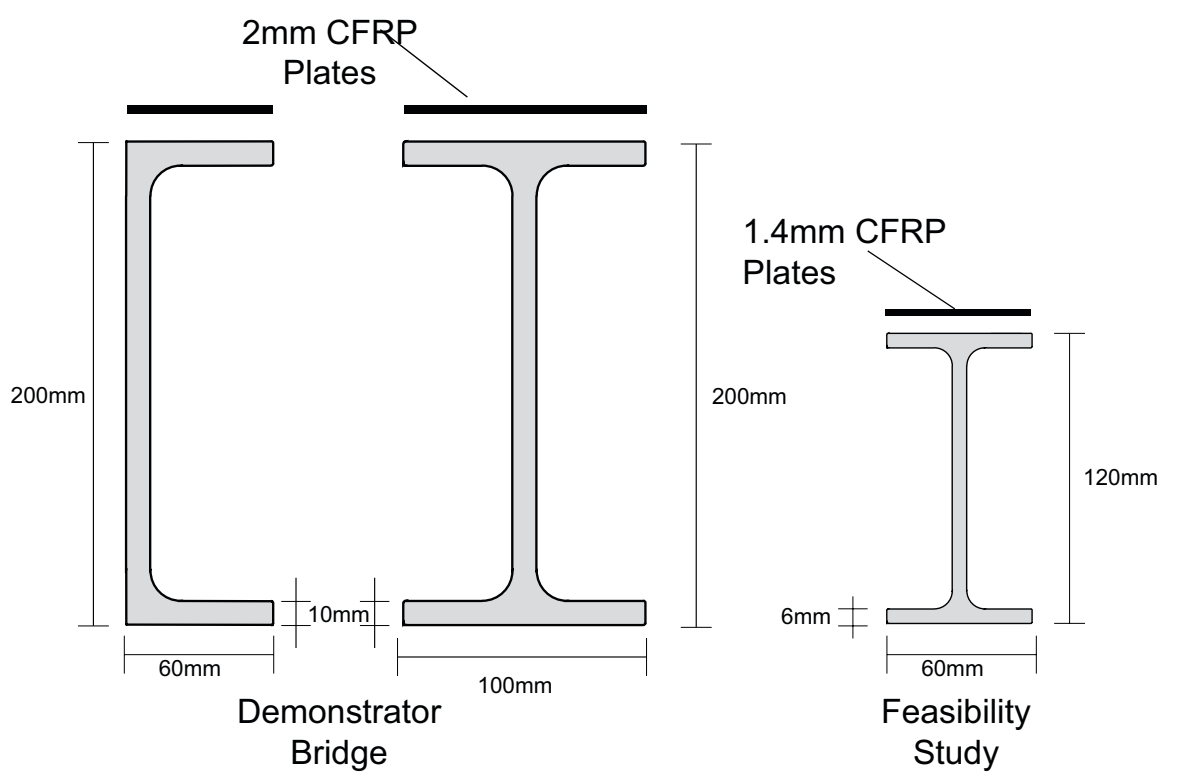

Figure 2. Comparison of the GFRP profile used for the described experiments with the profiles used to build Empa's demonstrator bridge. The CFRP plates that would be applied to the demonstrator are assumed to be slightly thicker.

One of the goals of the performed experiments was to confirm the ability to modify the stiffness of a sandwich structure with the initial properties of structural elements that are in use in actual engineering applications. The large dimensions of the sample used also made easier to carry out measurements to investigate the behavior under small dynamic loads. The obtained information was then used to estimate the effect of modification of the behavior of the structural element on the global behavior of Empa's demonstrator bridge.

Table 1. Main mechanical properties of the pultruded FRP profiles used in the demonstrator bridge and estimation of the effect of CFRP strengthening, based on (2). The CFRP plates are assumed to be as wide as sthe flanges and $2 \mathrm{~mm}$ thick, with an elastic modulus $\mathrm{E}=210 \mathrm{GPa}$. Shear properties $(G \cdot A$ estimated based on $G \approx 3000 M P a$.

\begin{tabular}{|l|c|c|c|c|} 
& I-profile, $D$ & I-profile, $G \cdot A$ & C-profile, $D$ & C-profile, $G \cdot A$ \\
\hline GFRP & $6.61 \cdot 10^{11} \mathrm{Nmm}^{2}$ & $5.4 \cdot 10^{6} \mathrm{~N}$ & $4.48 \cdot 10^{11} \mathrm{Nmm}^{2}$ & $5.4 \cdot 10^{6} \mathrm{~N}$ \\
GFRP+CFRP, not bonded & $6.61 \cdot 10^{11} \mathrm{Nmm}^{2}$ & $5.4 \cdot 10^{6} \mathrm{~N}$ & $4.48 \cdot 10^{11} \mathrm{Nmm}^{2}$ & $5.4 \cdot 10^{6} \mathrm{~N}$ \\
GFRP+CFRP, bonded & $15.0 \cdot 10^{11} \mathrm{Nmm}^{2}$ & $5.4 \cdot 10^{6} \mathrm{~N}$ & $9.5 \cdot 10^{11} \mathrm{Nmm}^{2}$ & $5.4 \cdot 10^{6} \mathrm{~N}$
\end{tabular}

\section{ADAPTIVE MODIFICATION OF THE BENDING STIFFNESS}

Next to the available strategies for the suppression of structural vibrations, such as the introduction of discrete passive or semi-active damping devices ${ }^{2,3}$ or even of active control systems, the modification of the mechanical properties of a structure offers a different approach to the solution of this problem. So far, the reduction of the amplitude of unwanted vibrations is obtained by extracting and dissipating mechanical energy from the system 
that has been excited by the environment. In most cases, extreme vibration amplitudes are due to (lock-in) resonance phenomena e.g. under the effect of wind or pedestrians walking on a bridge. Normally no influence can be taken on the forces exciting a structure to vibrate. This leads to the hypothesis that one could elude the onset of resonant vibrations if it were possible to modify at will the mechanical properties of a structure and thus its natural frequencies. The modification of the mechanical properties of a structure makes it possible to reject highly energetic components ${ }^{4}$ of an external excitation source in a targeted manner.

The realization of a structural element with adaptive mechanical properties implies either the ability to modify the elastic properties of the material the structure is made of or to modify the geometry of its cross section. According to (1) the bending properties of an element with constant cross section are:

$$
D=E I_{z}
$$

Where $D$ is the bending stiffness of the element, $E$ is the elastic modulus of the material the element is made of and $I_{z}$ is the second moment of the cross sectional area of the element under consideration.

The modification of $E$, can only be taken in consideration for a limited number of materials and is generally bound to phase transformations taking place at given temperatures. A modification of the bending properties by means of a change of elastic modulus is expected to be energy intensive and relatively slow because of the thermal processes involved.

The modification of $I_{z}$ via a change of the cross section geometry of a structure is generally also a very energy intensive process, if $E$ is constant and sufficiently high for the material to be used for structural applications.

In this work, the bending stiffness of a composite glass fiber reinforced polymer (GFRP) carbon fiber reinforced polymer I-beam is modified by coupling the stiffening elements made of carbon fiber reinforced polymer (CFRP) by means of friction stresses. The normal stresses between GFRP beam and CFRP stiffening plates that are necessary to generate friction stresses are obtained by applying an electrostatic field between beam and plates. The coupling causes the axis from which the second moment of area of the CFRP elements is calculated to shift by half the height of the beam thus increasing the total stiffness as follows:

$$
D_{\text {bonded }}=D_{\text {notbonded }}+2 \cdot A_{C F R P} \cdot E_{C F R P}\left(\frac{h}{2}\right)^{2}
$$

Where $D_{\text {bonded }}$ and $D_{\text {notbonded }}$ represent the stiffness of the multilayer beam with and without bond between layers, respectively. $\mathrm{h}$ is the sum of the heights of the GFRP beam and of the CFRP plates. $A_{C F R P}$ and $E_{C F R P}$ are the cross sectional area and the elastic modulus of a CFRP plate, respectively. Based on a bending stiffness of $71.3 \cdot 10^{9} \mathrm{Nmm}^{2}$ for the GFRP beam shown in figure 3 and $A_{C F R P}=84 \mathrm{~mm}^{2}$ (with $b_{C F R P}=60 \mathrm{~mm}$, $h_{C F R P}=1.4 \mathrm{~mm}$ and $E_{C F R P}=210 \cdot 10^{3} \mathrm{~N} / \mathrm{mm}^{2}$ ) we obtain:

Table 2. Main mechanical properties of the pultruded GFRP profile used for the described experiments and estimation of the effect of CFRP strengthening. Shear properties $(G \cdot A$ are estimated based on $G \approx 3000 M P a$

\begin{tabular}{|l|c|c|} 
& \multicolumn{1}{|c|}{$D$} & $G \cdot A$ \\
\hline GFRP & $7.13 \cdot 10^{10} \mathrm{Nmm}^{2}$ & $1.9 \cdot 10^{6} \mathrm{~N}$ \\
GFRP+CFRP, not bonded & $7.13 \cdot 10^{10} \mathrm{Nmm}^{2}$ & $1.9 \cdot 10^{6} \mathrm{~N}$ \\
GFRP+CFRP, bonded & $1.98 \cdot 10^{11} \mathrm{Nmm}^{2}$ & $1.9 \cdot 10^{6} \mathrm{~N}$
\end{tabular}

As expected, the contribution of the CFRP plates to the bending stiffness is negligible, when no bond between them and the GFRP beam is given. The ratio $D_{\text {bonded }} / D_{\text {notbonded }}$ is approximately 2.78 . If no contribution from shear deformations is considered, $\mathrm{t}$ he natural frequency of the beam can be expected to increase by a factor of approximately $1.7\left(\approx \sqrt{D_{\text {bonded }} / D_{\text {notbonded }}}\right)$, when stiff CFRP plates are applied to its surface. The shear stiffness $G \cdot A$ was estimated on the base of a shear modulus of GFRP $(G \approx 3000 M P a)$ and under the assumption that the shear stiffness is solely determined by the web of the I-beam as it is often assumed for sandwich beams.

First calculations indicate that an increase by a factor of 2 of the bending stiffness of the longitudinal beams in the bridge deck (see figure 1,right hand side) the first bending eigenfrequency of the deck supported by two 
stay cables (instead of 6 as in the figure) can be shifted by $13.9 \%$ from $5.77 \mathrm{~Hz}$ to $6.57 \mathrm{~Hz}$, if the shear deformations of the beam are neglected. A more remarkable modification of the first bending eigenfrequency is expected if an individual section of the deck is taken in consideration (from $29 \mathrm{~Hz}$ to $41 \mathrm{~Hz}, \Delta \%=41.4 \%$ )

\section{EXPERIMENTAL SETUP}

In the set up used for the experiments, the bond between additional stiffening elements (CFRP plates, CarboDur M614, Sika, CH) and the core of the sandwich beam (GFRP I-beam, Fiberline Composites, DK) is given by electrostatic forces generated by an electrical field built up across a $0.08 \mathrm{~mm}$ thick Polyvinylidenefluoride (PVDF, $\epsilon_{r} \approx 2$, CS Hyde Company, USA) film. The PVDF film was applied to the flanges of the GFRP beam using silver loaded epoxide resin (Eccobond 57C, Emerson Cuming, USA) that served at the same time as electrode, thank to its good electric conductivity and as adhesive. The CFRP plates served at the same time as second pair of electrodes and stiffening member. This setup was chosen in order to optimize the electrostatic forces that could be obtained per unit voltage.

For comparison, the behavior of an identical system, in which the CFRP plates are bonded to the GFRP using a common epoxide type adhesive (Araldite AW 106 and HV 953 U, Huntsman, B) was observed, in order to determine the efficiency of the electrostatic coupling of the CFRP elements.

The multilayer beams were fixed at one end and excited at the free end by means of the electromagnetic fields generated by a coil, acting on a $40 \mathrm{~mm} \times 40 \mathrm{~mm} \times 20 \mathrm{~mm}$ FeNdB permanent magnet that was attached to the free end of the beam, $2240 \mathrm{~mm}$ from its fixation point. The brackets visible in the left hand side of Figure 3 were only used to keep the loose CFRP plates in place when the electrostatic field was turned off, ideally without exerting any normal forces. Two additional masses (1050 g each) were placed $2090 \mathrm{~mm}$ (position of the center of gravity) from the fixed end of the beam to separate the first bending and torsion modes.

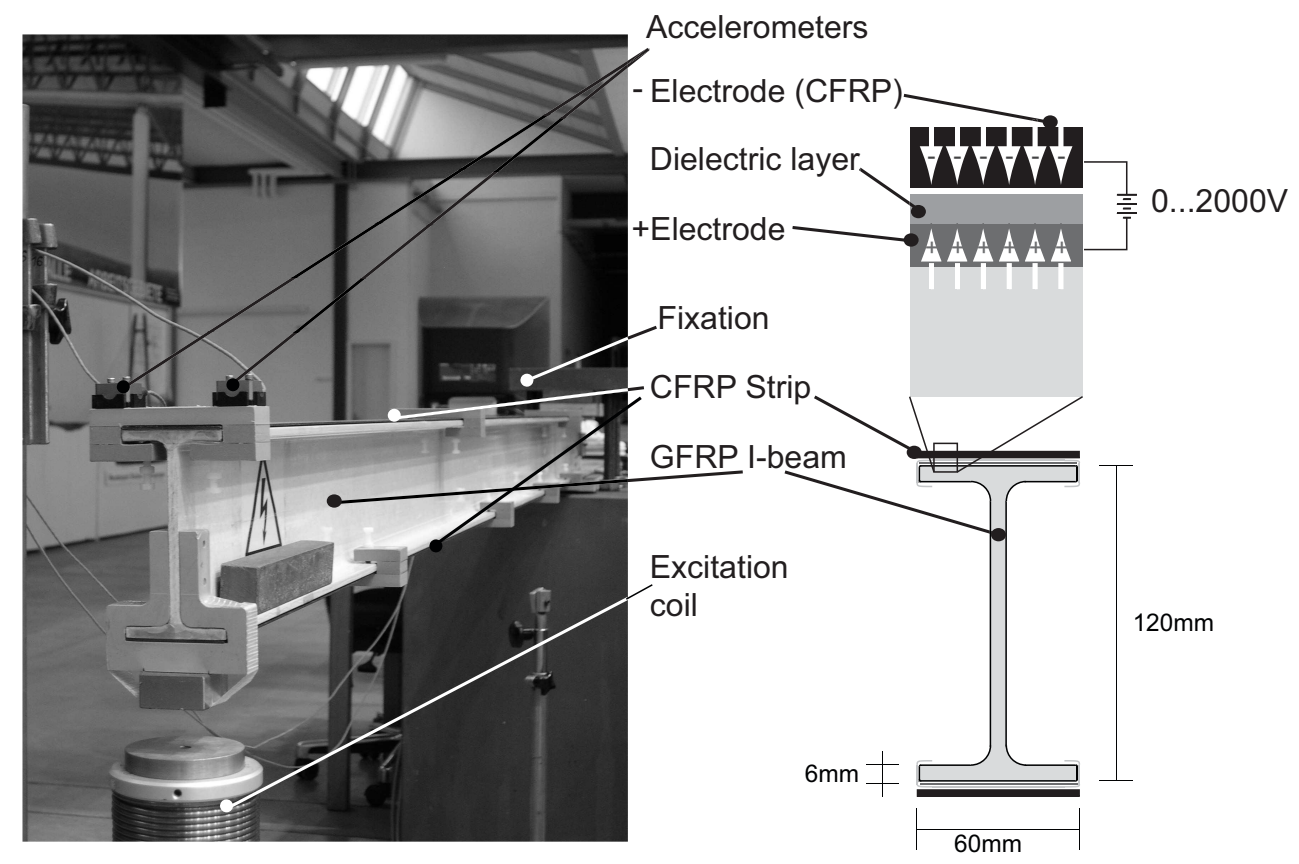

Figure 3. Overview of the test set-up and cross section of a GFRP I beam as used in the experiments described in section 4

The currents required to induce the exciting alternate magnetic field, were generated by a bipolar operational power amplifier (BOP 20-20M, Kepco, USA) driven in its current controlled mode by an arbitrary signal generator (33120 A, Agilent, USA). 
The transversal accelerations at the end of the composite beam were measured by means of two accelerometers (PCB Piezotronic, Mod. 3701G3FA3G, with a sensitivity of $1 \mathrm{~V} / \mathrm{g}$ ) positioned at the left and the right edges at the free end of the beam (sensor 1 and sensor 2, respectively). The acceleration and the current circulating through the coil were measured with a sampling frequency of $512 \mathrm{~Hz}$ using a digital analog data acquisition board (6036 E DAQ, National Instruments, USA). The components of the sandwich beam were connected to a high voltage power supply (PS 350, Stanford Research Systems, USA), so that the necessary electrical potential $U_{i}$ could be applied between faces and core. The complete test setup was controlled from a PC via a LabView interface developed for this purpose.

\section{MEASUREMENTS AND RESULTS}

The beam was excited with a $7-17 \mathrm{~Hz}$ linear chirp signal at a $1.2 \mathrm{~A}$ peak to peak current amplitude. The vertical accelerations of the beam end and the coil current (assumed to be directly proportional to the generated electromagnetic-field, hence to the force exerted on the magnet at the end of the beam) were recorded as a function of time. Double integration of the acceleration data yielded the displacements at the end of the beam.

From the current and the displacement data (average of sensor 1 and sensor 2), transfer functions of the system were calculated for different $U_{i}$ potential levels, as shown in figures 4 . The figures show the behavior of the system for interlaminar potentials between $0 \mathrm{~V}$ and $2000 \mathrm{~V}$ as well as for the not bonded and fully bonded cases. In not bonded case, the CFRP plates were laid on the GFRP beam (respectively held against it) but no PVDF layer was inserted between them. In the bonded case, the CFRP and GFRP elements were joined with the epoxy type adhesive.

A quite remarkable reduction of the vibration amplitude of the beam (approximately $15 \mathrm{~dB}$ in the transfer function) can be noticed between the not bonded beam and the $0 \mathrm{~V}$ case, which is probably due to a different friction coefficient of the CFRP-GFRP and PVDF-CFRP material pairs. A reduction of additional 20 to $15 \mathrm{~dB}$ is observed as the potential $U_{i}$ is increased to $400 \mathrm{~V}$ and $800 \mathrm{~V}$, respectively. The maximum of the transfer function increases again (to levels comparable to $0 \mathrm{~V}$ ) at $U_{i}=2000 \mathrm{~V}$ and again for the fully bonded CFRP plates.

As expected, the eigenfrequency, as determined from the phase discontinuity in the plot on the right hand side, does not increase in a measurable way for the not bonded system in comparison to the system at $U_{i}=0 \mathrm{~V}$. The application of the interlaminar voltage $U_{i}=400 \mathrm{~V} \ldots 2000 \mathrm{~V}$ increases the normal stresses at the contact surface between the PVDF dielectric layer and the CFRP plates, thus increasing the stiffness of the system and its natural frequency. Finally, the maximum effect for a given geometry and material properties is given by a full bond between CFRP and GFRP elements. This is obtained by joining the elements with the epoxy adhesive.
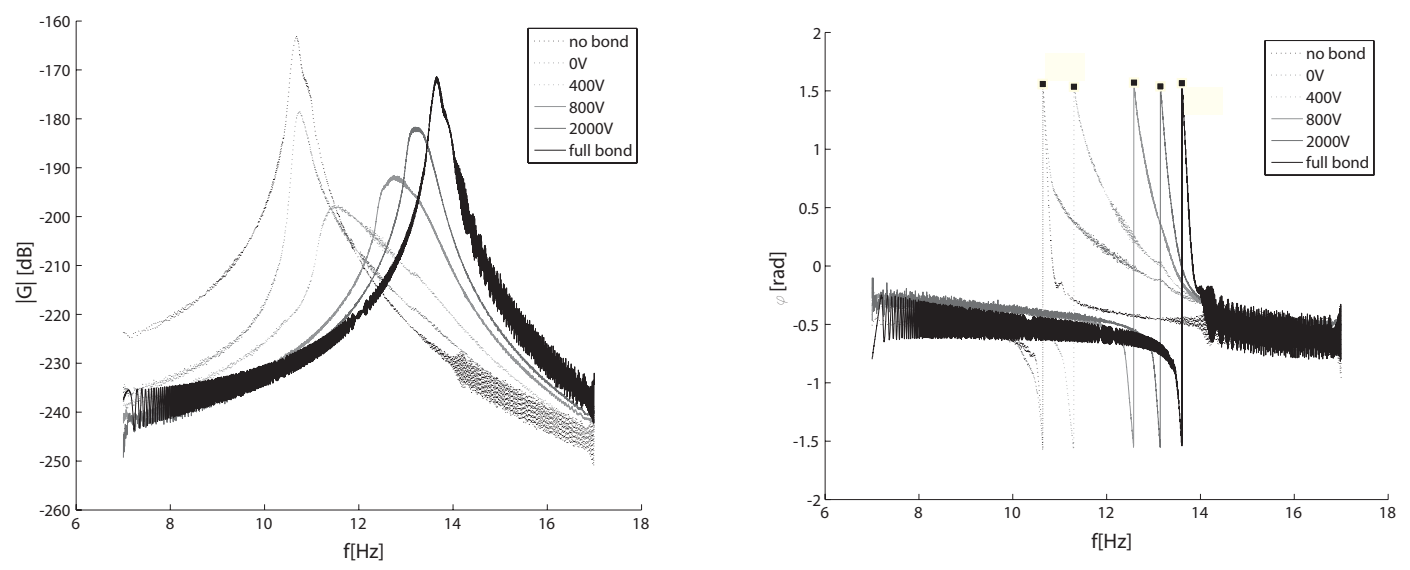

Figure 4. The change of the transfer function (amplitude on the left hand side, phase on the right hand side) measured at different $U_{i}$ levels indicates a change in the mechanical behavior of the system. Excitation current: $1.2 \mathrm{~A}$ pp 
The efficiency $\eta$ of the stiffening by means of the CFRP plates is considered to be 1 when the plates are joined to the I-beam with epoxy adhesive.

$\eta$ is a measure of the efficiency of the coupling of the additional CFRP plates in shifting the natural frequency $f_{0}$ of the beam and is calculated as:

$$
\eta\left(U_{i}\right)=\frac{f_{0}\left(U_{i}\right)-f_{0}(\text { nobond })}{f_{0}(\text { fullbond })-f_{0}(\text { nobond })}
$$

$\eta$ is an indirect measure of the quality of the bond obtained by the electrostatic coupling of CFRP and GFRP elements. Coupling by means of a conventional resin is considered to yield the best possible results. Electrostatic coupling is then compared to such a situation.

The resonance frequencies $f_{0}$ are identified from the phase plots and are summarized in table 3 .

The quality of the system as a resonator is described by the q-factor:

$$
q=\frac{f_{0}}{f_{1}-f_{2}}=\frac{f_{0}}{\Delta f}
$$

Where $f_{0}$ is the resonance frequency and $f_{1}$ and $f_{2}$ are the frequencies at which the transfer function has values $6 \mathrm{~dB}$ lower than at $f_{0}$. A high q-factor indicates that the resonator oscillates at a given frequency with a narrow bandwidth. q-factors for the different configurations are listed in table 4

Table 3. Resonance frequency shift as a function of the bonding state of the CFRP plates to the GRFP beam. $\Delta$ and $\eta$ calculated using the values for the not bonded case as a reference.

\begin{tabular}{|c|c|c|c|c|}
$U_{i}[\mathrm{~V}]$ & $f_{0}[\mathrm{~Hz}]$ & $\Delta[\mathrm{Hz}]$ & $\Delta \%$ & $\eta$ \\
\hline no bond & 10.64 & - & - & 0 \\
$0 \mathrm{~V}$ & 10.64 & 0 & $0 \%$ & 0 \\
$400 \mathrm{~V}$ & 11.30 & 0.66 & $6.2 \%$ & 0.22 \\
$800 \mathrm{~V}$ & 12.58 & 1.94 & $18.2 \%$ & 0.66 \\
$2000 \mathrm{~V}$ & 13.15 & 2.51 & $23.6 \%$ & 0.87 \\
full bond & 13.6 & 2.97 & $27.9 \%$ & 1
\end{tabular}

Table 4. q-factors calculated according to (4)

\begin{tabular}{|c|c|c|c|}
$U_{i}[\mathrm{~V}]$ & $f_{0}[\mathrm{~Hz}]$ & $\Delta f[\mathrm{~Hz}]$ & $q$ \\
\hline no bond & 10.64 & 0.22 & 48.4 \\
$0 \mathrm{~V}$ & 10.64 & 0.31 & 34.3 \\
$400 \mathrm{~V}$ & 11.30 & 1.20 & 9.4 \\
$800 \mathrm{~V}$ & 12.58 & 0.94 & 13.4 \\
$2000 \mathrm{~V}$ & 13.15 & 0.53 & 24.8 \\
full bond & 13.6 & 0.28 & 48.6
\end{tabular}

\section{CONCLUSIONS AND OUTLOOK}

The transfer functions of the beam at different $U_{i}$ values suggest that two mechanisms contribute to the modification of the mechanical behavior of the system:

- The shift in resonance frequency $f_{0}$ indicates that the electrostatic coupling of the CFRP plates increases the stiffness of the system. 
- The broadening of the resonance peaks, especially for intermediate voltages, indicates that increased damping is introduced in the system.

This situation can be interpreted as the plates adhering to the surface of the beam and as shear stresses being transferred across the interface effectively, as long as the stresses do not exceed a certain level. At higher deformation values, the shear stresses at the interface exceed the maximum stress that can be transferred at the interface by adhesion and a slip mechanism is activated. At this point energy is dissipated at the interface by friction.

At the highest voltage tested $\left(U_{i}=2000 \mathrm{~V}\right)$, the shear stresses transferred at the interface are sufficient to obtain a behavior closer to the behavior of an elastic body, thus yielding a higher resonance frequency and a higher q-factor than at intermediate voltage levels.

The comparison of the transfer functions measured on the electrostatically coupled GFRP-CFRP system with the transfer function of the adhesive coupled system gives an indication of the efficiency of electrostatic coupling in comparison with joining of the elements of the system. While a $100 \%$ efficiency (i.e. the stiffening obtained by joining all elements with an adhesive) in the tuning of the resonance frequency through electrostatic coupling of the additional CFRP elements cannot be achieved with the used dielectric system, the $87 \%$ value achieved with the current system is a very encouraging result.

As opposed to previous work ${ }^{5}$ where materials with very low elastic moduli were used to demonstrate the effect of the electrostatic coupling of stiffening elements to the soft core of a sandwich structure, in this work commercially available structural elements were utilized. The obtained results indicate that also the stiffness of such structural elements can be successfully modified, thus opening the way to the development of lightweight adaptive structures.

As expected, the low shear stiffness of the web has a strong effect on the vibratory behavior of the I beam that reduces the efficiency of the application of stiffening elements in terms of frequency shift. One of the goals of

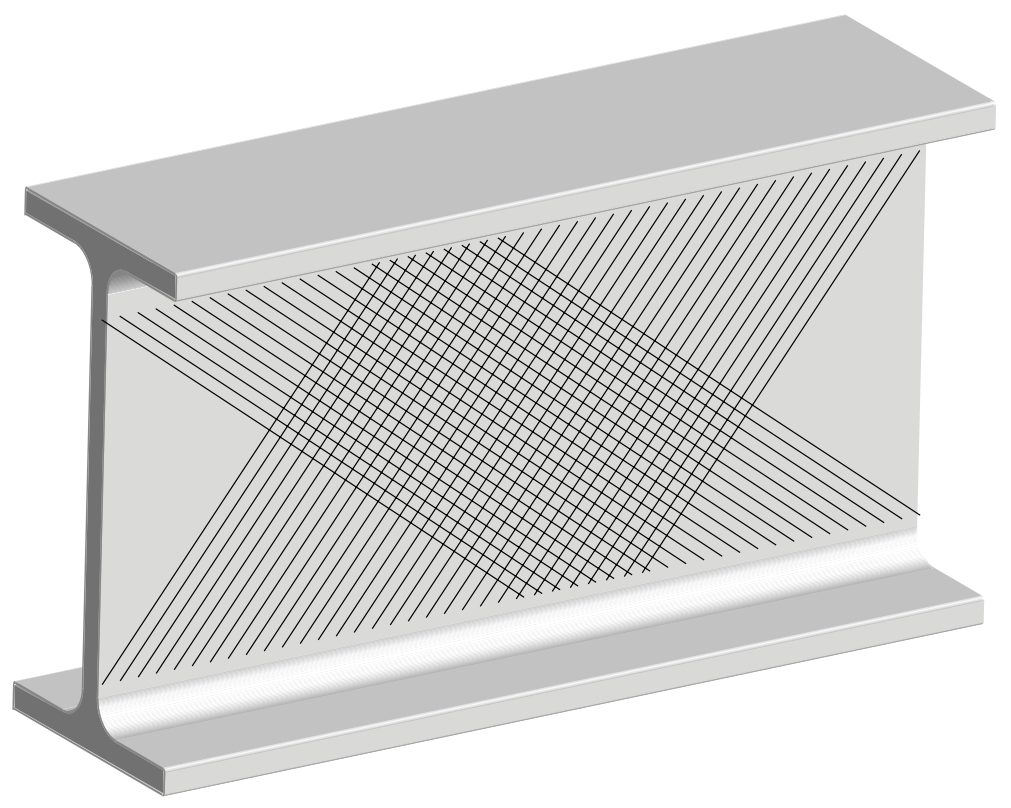

Figure 5. Application of adhesively or electrostatically bonded $\pm 45^{\circ}$ crossplies can be used to increase the shear stiffness of the web, thus increasing the efficiency of the electrostatic tuning of the bending stiffness

the presented work was to assess the feasibility of the tuning of the natural frequencies of Empa's Demonstrator Bridge by means of the electrostatic coupling of stiffening elements to the structure. Based on the obtained results (maximum frequency shift of $23.6 \%$ for an individual beam), a moderate effect can be expected to be 
seen in the complete structure. In a structural element with a higher shear stiffness, a stronger effect in terms of frequency shift can be expected.

In order to counter the effect of the low shear stiffness of the beam, stiffening (permanent or adaptive) would also be required, in order to obtain a stronger effect. In figure 5 a possible approach to the shear stiffening of the web, consisting of $\pm 45^{\circ}$ carbon fiber crossplies, is sketched. Given the geometry of the beam, an electrostatically couplable version of the system will be considered. The application of adaptive bending and shear stiffening would provide with a greater degree of freedom in the modification of the behavior of the bridge deck.

Future work concerning the adaptive modification of the bending stiffness of the deck of Empa's Demonstrator bridge, will be focused on the optimization of the dielectric system used for the electrostatic coupling of the CFRP elements on one hand and on the implementation of a shear stiffening system for the web of the GFRP beams.

\section{ACKNOWLEDGMENTS}

The authors wish to thank Dr. Daniel Gsell of Empa's Structural Engineering Research Laboratory for his kind support and for the calculations performed to estimate the effect of the stiffening of selected beams on the global behavior of the structure under consideration.

\section{REFERENCES}

1. D. Gsell and M. Motavalli, "Indoor cable stayed gfrp-bridge at empa, switzerland," in 4th International Conference on Advanced Composite Materials in Bridges and Structures, (Calgary, AL, Canada), 2004.

2. F. Nitzsche and T. e. a. Harold, "Development of a maxium energy extraction control for the smart spring," Journal of Intelligent Material Systems and Structures 16, pp. 1057-1066, November/December 2005.

3. F. Weber, G. Feltrin, and M. Motavalli, "Passive damping of cables with mr dampers," Journal Material and Structures 38, pp. 595-600, June 2005.

4. R. D. Kornbluh, H. Prahlad, R. Pelrine, S. Stanford, M. A. Rosenthal, and P. A. von Guggenberg, "Rubber to rigid, clamped to undamped: toward composite materials with wide-range controllable stiffness and damping," in Proceedings of the SPIE - The International Society for Optical Engineering, vol.5388, no.1, pp. 372-386, 2004. Conference Paper.

5. A. Bergamini, R. Christen, and M. Motavalli, "Electrostatic tuning of the bending stiffness of simple slender multilayer composite structures," in Proceedings of the SPIE - The International Society for Optical Engineering, vol.5760, pp. 152-163, SPIE - The International Society for Optical Engineering, (San Diego, Ca), 2005. Conference Paper. 